\title{
„Der dich erhält, wie es dir selber gefällt.“
}

\section{Transidentität als Ernstfall Systematischer Theologie}

\begin{abstract}
The positive value of diversity across the range of Queer Theology is portrayed less concretely in systematic designs of newer theological classifications that focus on freedom and acknowledgement; however, thus far the information in Queer Theology has seldom covered great systematic-theological designs of the present, resulting in important tools for greater understanding going unnoticed. At the same time, in theology and religion there is a distinct lack of material that addresses trans identities and the many forms of otherness. Few exceptions exist where these issues are discussed. It shall be proven here that systematic theology in particular has considerable potential for a non-sensationalist assessment of otherness. Moreover, a theology that applies the paradigm of acknowledgement argues inclusively, not exclusively. Continuing on from Marcella Althaus-Reid and Susannah Cornwall's Queer Theology, Falk Wagner and Eberhard Jüngel's theories on theological acknowledgement raise the question of whether or not "Who keeps you the way you yourself decided to be" is also applicable for trans people.
\end{abstract}

Ist Anderssein Charakteristik des Menschen, dann stellen Modi des Andersseins keineswegs Sensationelles dar. Sind Ambiguitäten menschlicher Körper und psychischer Verfasstheit notorisch, dann missversteht man das Seltene als Devianz. Entspricht die anthropologische Wirklichkeit keinem bloß Vorurteile reflektierenden Essentialismus, ${ }^{1}$ der als deterministischer Naturalismus verstanden und ausgeschlossen werden kann, ${ }^{2}$ dann handelt es sich bei menschlicher Vielfalt um kategorisch nicht zu unterscheidende Diversifikationen. Zwar ist in Geltung, dass Bewusstheitsveränderungen keineswegs auf Gewusstseinsveränderungen folgen müssen, aber immerhin kann argumentativ das Nicht-Sensationelle des Vielen und Anderen bewährt werden. Dies gilt auch für Transsexualität, die viel ad-

1 Vgl. Jörg Y. Fehige, „Transsexualität zwischen Genetik und sozialer Praxis,“ Deutsche Zeitschrift für Philosophie, Bd. 57, 2009, $757-780$.

2 Vgl. Magnus Striet, „Fortschritt und Kontingenz. Ein Beitrag zur Enhancementdebatte,“ in Entgrenzung des Menschseins? Eine christliche Antwort auf die Perfektionierung des Menschen, hg. von Gebhard Fürst und Dietmar Mieth, Paderborn et al.: Schöningh 2012, 131-139, 133. 
äquater als Transidentität gefasst wird, da geschlechtliches Empfinden nicht einfach äußerlich bleibt oder lediglich partielle Bedeutung hat. ${ }^{3}$

Wenn Livia Prüll, eine Transfrau und Medizinhistorikerin, den Mut aufbringt, gegen den oft mit kleinbürgerlichem Material gefüllten Mainstream das Fluidale des Geschlechtlichen zu leben und dazu auf das bekannte Kirchenlied von Joachim Neander (1650 -1680), Lobe den Herren (EG 317), rekurriert, wo es in der dritten Strophe heißt: „der dich erhält, wie es dir selber gefällt““4, dann kann das Plädoyer gegen eine Sensationalisierung transidenten Fühlens kaum überhört werden. Da Prüll hier an das christliche Ambiente einer von Gott getragenen Freiheit erinnert, die die unwiederholbare Einmaligkeit von Transpersonen und aller Menschen in ihrem Eigensein keineswegs gefährdet, ${ }^{5}$ sondern ermöglicht, ordnet sie Transempfinden dem Optionsraum zu, den Menschen notorisch nutzen, um zu werden, was sie sein wollen. Entgegen dem schlechten Ruf, den ein Individualismus im Christentum hat, und gegen den latenten ironisch-denunziatorischen Ton eines Vulgärchristentums, das immer schon zu wissen meint, was gelungene Modalitäten menschlichen Lebens sind, erinnert Prüll an das NichtSensationelle der viel zu oft starr gedachten Geschlechtlichkeit des Menschen als eines Beispiels für die Mannigfaltigkeit des Menschseins und Selbstseins. Luthers „Hier stehe ich, ich kann nicht anders“ figuriert dabei als ermutigendes Beispiel einer Psychologie des Selbstseins. ${ }^{6}$

3 Vgl. Udo Rauchfleisch, Transsexualität - Transidentität. Begutachtung, Begleitung, Therapie, 5. Aufl., Göttingen: Vandenhoeck \& Ruprecht 2016 [2006], 26 und Mathias Wirth, „Living in a Shell of Something I'm Not.“ Transsexuality, Bioethics and the Judeo-Christian Perspective, “Journal of Religion \& Health, Bd. 54, 2015, 1584-1597, 1585 - 1586, sowie Mathias Wirth, „Was bedeutet: Unbedingte Anerkennung der Andersheit des Anderen? Intersexualität und Transidentität im Licht advokatorischer Ethik,“ in Inter* und Trans*identitäten. Ethische, soziale und juristische Aspekte, hg. von Maximilian Schochow, Saskia Gehrmann und Florian Steger, Gießen: Psychosozial-Verlag 2016, 105-133, $112 \mathrm{f}$.

4 In der autobiographischen Einführung ihres Vortrags auf einer Tagung zu Trans und Inter an der Universitätsklinik Halle (Saale) 2014 hat Prüll auf die persönliche Bedeutung dieses Verses hingewiesen, vgl. ihren entsprechenden Vortrag: Livia Prüll, „Das Selbstbild der transidenten Frau nach 1945 und die Konsequenzen für den Umgang mit Geschlechtsidentitäten,“ in Inter* und Trans*identitäten. Ethische, soziale und juristische Aspekte, op. cit., 33 -56. Vgl. auch Livia Prüll, Trans* im Glück. Geschlechtsangleichung als Chance. Autobiographie, Medizingeschichte, Medizinethik, Göttingen: Vandenhoeck \& Ruprecht 2016, 9 f.

5 Vgl. Mathias Wirth, „Eigensinn gefährdet die Güte Gottes nicht. Der Prophet Jona und eine alttestamentliche Kritik des Gehorsams, “ Kerygma und Dogma, Bd. 60, 2014, 169-189.

6 Vgl. Gesa Lindemann, Das paradoxe Geschlecht. Transsexualität im Spannungsfeld von Körper, Leib und Gefühl, 2. Aufl., Wiesbaden: Verlag für Sozialwissenschaften 2011 [1993], 82. Auf dieses Diktum Luthers nehmen auch Protagonisten der Queer Theology Bezug, vgl. Elizabeth Stuart et al., 
Dieser Beitrag erörtert die Frage, inwiefern die christliche Theologie zu einer nicht-sensationellen Interpretation der Transidentität beitragen kann. Außerdem steht eine solche Theologie gegen den Verdacht, ihre Rede vom Anderen und Vielen traktiere lediglich einen Purismus des bloß abstrakten Anderen, der weder provoziert noch Raum zum Selbstsein einfordert. Gegen diesen Verdacht einer Blutleere der Theologie soll zuerst die vornehmlich aus dem englischsprachigen Raum stammende Queer Theology eingeführt werden, der das Verdienst zukommt, sexuelle und geschlechtliche Vielfalt zum theologischen Thema gemacht zu haben, die allerdings dazu neigt, teilweise expressis verbis, der Andersheit sexueller Vielfalt einen Platz in der Mitte der Gesellschaft auszureden, so aber den diskriminierenden Sensations-Charakter der Transidentität substantiiert (I.). ${ }^{7}$ Dann soll Transidentität als theologische Nicht-Sensation besprochen werden, indem oft im Allgemeinen verbleibende Theologien der Freiheit und Anerkennung konkretisiert werden (II.). Erscheint Transidentität dabei als konkreter Anspruch des Anderen und als unbedingte Forderung der Freiheit nach Achtung, dann gibt es keinen Grund, Transpersonen im Abseits des Abnormen oder Enormen zu sehen. Darauf werden neuere systematisch-theologische Ansätze eingeführt, die der Subjekthaftigkeit und Mündigkeit des Menschen positive Aufmerksamkeit geschenkt haben, wie Falk Wagner (II.1) und Eberhard Jüngel (II.2). ${ }^{8}$ Transidentität soll dabei

Religion is a Queer Thing. A Guide to the Christian Faith for Lesbian, Gay, Bisexual and Transgendered People, London und Washington: Cassell 1997, 19.

7 Vgl. Marcella Althaus-Reid, Lisa Isherwood, „Thinking Theology and Queer Theory,“ Feminist Theology, Bd. 15, 2007, 302-314, 304.

8 Aus Gründen der Konzentration werden nur zwei evangelisch-theologische Ansätze diskutiert. Leicht wäre auch die Freiheitsanalyse des katholischen Dogmatikers Thomas Pröpper auf das Anliegen der Anerkennung von Trans anwendbar gewesen. Es wäre besonders an seine Ausführungen zum Begriff der formal unbedingten Freiheit anzuknüpfen. Hierbei geht es um die Fähigkeit, sich zu allem und jedem in Distanz setzen zu können, explizit auch zu „unmittelbaren Gegebenheiten“. Im Distanzieren geschieht Überschreiten und Reflexion, wie Pröpper betont, an dessen Ende Affirmation oder Negation zum Objekt der Distanz steht, vgl. Thomas Pröpper, Theologische Anthropologie, Teilbd. 1, 2. Aufl., Freiburg i.Br.: Herder 2012 [2011], 501 und dazu Striet, „Fortschritt und Kontingenz,“ 132. In dieses Schema der Überschreitung durch Distanzierung, die den Menschen nicht einfach an das Vorgefundene fixiert, lässt sich leicht das Begehren und Verhalten von Trans einordnen, nicht zuletzt, weil Freiheit hier als Selbstbestimmung und Selbstentwurf begriffen wird, was ausdrücklich auch den Körper betrifft, vgl. Pröpper, Theologische Anthropologie, $503 \mathrm{f}$. und ähnlich Striet, „Fortschritt und Kontingenz,“ 134 sowie Ludger Honnefelder, „Perfektionierung des Menschen? Paradigmen, Ziele und Grenzen,“ in Entgrenzung des Menschseins? Eine christliche Antwort auf die Perfektionierung des Menschen, op. cit., 13-26, 13-17. Zum begonnenen Gespräch zwischen Pröppers Freiheitsanalyse und Transidentität, vgl. Wirth, „Was bedeutet: Unbedingte Anerkennung der Andersheit des Anderen?,“ 126. 
als Testfrage für die Reichweite von Anerkennungstheoremen in der Theologie fungieren. Im abschließenden Fazit wird ein differenzempfindlicher Konkretismus am Beispiel der Transidentität als Ernstfall Systematischer Theologie vorgeschlagen, die oft viel zu abstrakt den unbedingten Anspruch des Anderen und der Freiheit zum Sinnhorizont der Praxis erklärt hat (III.).

\section{Transidentität als theologische Sensation: Queer Theology}

Im Zuge der Verschärfung des Bewusstseins für sexuelle und geschlechtliche Vielfalt ${ }^{9}$ hat insbesondere eine aus der Tradition der Befreiungstheologie und der Feministischen Theologie stammende Systematik Arbeit an den entsprechenden Vakanzen in der Theologie begonnen und Themen wie Homosexualität, Intersexualität und Transsexualität zu Themen von Rang erhoben. ${ }^{10}$ Dabei ist es besonders dem Pioniergeist der viel zu früh verstorbenen Marcella Althaus-Reid zu verdanken, durch eine vielfach anstößige Theologie eine Brücke über den Riss zwischen Theologie und all denen gebaut zu haben, die sich nicht an das heteronormativ-dichotome Konstrukt von Geschlechtlichkeit und Sexualität anschmiegen. Auch wenn man zugibt, dass einige der Thesen Althaus-Reids kaum konsensfähig sein dürften, ist auch mit ihrem Namen das Projekt einer Queer Theology verbunden, die weit mehr sein will als Thematisierung von LGBTI-Anliegen im Raum der akademischen Theologie. Das Anliegen einer Trans-Formation der ganzen Theologie durch queer-Paradigmen verdient dabei aufmerksame Diskussion, der sich mittlerweile eine Vielzahl von Einzelstudien gewidmet hat. Neben Althaus-Reid ist es Susannah Cornwall von der Universität Exeter in Großbritannien, die sich innerhalb einer kontextuellen Theologie auch für Queer Theology interessiert und viel beachtete Arbeiten dazu vorgelegt hat.

Bei der folgenden (kritischen) Inspektion sollen ethische Errungenschaften der kritischen Methode des heterogenen Spektrums einer Queer Theology für die durch hegemoniale Sexualitäts- und Geschlechterkonzepte Diffamierten keineswegs gering geschätzt werden. Allerdings stellt sich die Frage, ob eine eigene Queer Theology nicht den Graben durch Sensationalisierung weiter vertieft, den sie

9 Vgl. Isolde Karle, „Da ist nicht mehr Mann noch Frau...“. Theologie jenseits der Geschlechterdifferenz, Gütersloh: Gütersloher Verlagshaus 2006, 255.

10 Vgl. Claudia Schippert, „Queer Theory and the Study of Religion,“ Revista de Estudos da Religião, Bd. 4, 2005, 90-99; Melissa M. Wilcox, „Outlaws or In-Laws? Queer Theory, LGBT Studies, and Religious Studies,“ Journal of Homosexuality, Bd. 52, 2006, 73 -100, 91. 
wenigstens teilweise ebnen wollte, nun aber nicht das Gemeinsame, sondern das Unterscheidende betont, ohne das Unterscheidende als anthropologische Konstanz auszuweisen.

\section{Queer Theology bei Althaus-Reid}

Marcella Althaus-Reid hat sich deutlich gegen den Verdacht verwahrt, ihr Interesse an Theologie, Sexualität und Geschlecht stelle einen Verrat an ihrem ursprünglichen Engagement für eine theologische Option der Armen dar. ${ }^{11}$ Dagegen hat sie eingewendet, Befreiungstheologie sei nicht auf einzelne Themen beschränkt, sondern stelle einen theologischen Stil dar, ${ }^{12}$ dem es darauf ankäme, Befreiung auch in anderen Bereichen als denen ökonomischer Unterdrückung zu diskutieren. Sie nennt Transvestiten, mittellose Transgender, psychisch schwer Erkrankte und Obdachlose in den Vororten großer Städte, die Aufmerksamkeit der Befreiungstheologie benötigen. ${ }^{13}$

Die größte Kritik an der Theologie durch die Theologie sei aber von der Feministischen Theologie ausgegangen, die, so Althaus-Reid, den Gesamtbereich der Theologie unter Ideologieverdacht gestellt und mit der Analyse und Destruktion der Ideologie des Patriarchats und des androzentrischen Weltbildes in biblischen Schriften, Lehrentwicklungen und Theologien begonnen hat. Ihre Fundamentalopposition gegen eine virilitätsgeprägte Religion hat sie, so AlthausReid weiter, eine noch deutlichere Zurückweisung durch das theologische Establishment erfahren lassen, weil Genderdiskurse anthropologische und moralische Konventionen noch stärker erschütterten als Diskurse über gerechte Güterverteilung. Obwohl das Subversive mittlerweile Teil der theologischen Methodik sei,

11 Vgl. Althaus-Reid / Isherwood, „Thinking Theology and Queer Theory,“ 313. Dieser Vorwurf ignoriert besonders Althaus-Reids Betonung des bourgeoisen Kontextes westlicher Theologie (vgl. a.a.O., 302), den sie mit der Gruppe der Queer People konfrontiert, deren vielfach verweigerter Platz in der Mitte der Gesellschaft (inter hominem esse) keine Petitesse oder ein Luxusproblem saturierter Gesellschaften darstellt, vgl. Wirth, „Was bedeutet: Unbedingte Anerkennung der Andersheit des Anderen?,“ 122. Auch Jürgen Moltmann hat, ähnlich wie Hannah Arendt, von einem „sozialen Niemandsland“ gewarnt, in das Menschen geraten können, vgl. Jürgen Moltmann, „Gerechtigkeit für Opfer und Täter, “ in In der Geschichte des dreieinigen Gottes. Beiträge zur trinitarischen Theologie, München: Kaiser 1991, 74-89, 88.

12 Vgl. auch Falk Wagner, Zur Revolutionierung des Gottesgedankens. Texte zu einer modernen philosophischen Theologie, aus dem Nachlass hg. von Christian Danz und Michael MurrmannKahl, Tübingen: Mohr Siebeck 2014, 595-597.

13 Vgl. Marcella Althaus-Reid, From Feminist Theology to Indecent Theology. Readings on Poverty, Sexual Identity and God, London: SCM 2004, 148. 
blieben Armut und Sexualität die großen theologischen Tabus. ${ }^{14}$ Weil Sexualität aber Thema der Queer Theology ist, muss sie als Indecent Theology erscheinen, die das genaue Gegenteil von vornehm-zurückhaltend und domestiziert abgibt. ${ }^{15}$

Queer Theology in der Tradition der Befreiungs- und Feministischen Theologie $^{16}$ unternimmt eine kritische Inspektion der gesamten Theologie, die vielfach geschlechts-dichotom und hetero-normativ durchmachtet scheint. ${ }^{17}$ Dabei stellen sich auch solche Facetten heraus, die dem queer-Paradigma entsprechen, wie Jesu unbefangener Umgang mit Frauen ${ }^{18}$ oder die paulinische Eschatologie einer Überwindung des irdischen Geschlechts im Reich Gottes. ${ }^{19}$ Ethischer Maßstab

14 Vgl. a.a.O., 3 f. Dagegen spricht ein zunehmendes Interesse innerhalb der akademischen Theologie an einer queer-theory, vgl. Susannah Cornwall, Controversies in Queer Theology, London: SCM 2011, 187.

15 Vgl. Marcella Althaus-Reid, Indecent Theology. Theological Perversions in Sex, Gender and Politics, London und New York: Routledge 2000, 4-6, 195 f. An anderer Stelle hat Althaus-Reid das Programm der Indecent Theology pointiert, vgl. Marcella Althaus-Reid, „The Bi/girl Writing: From Feminist Theology to Queer Theologies,“ in Post-Christian Feminisms. A Critical Approach, hg. von Lisa Isherwood und Kathleen McPhillips, Aldershot: Ashgate 2008, 105-116, 107 : „Indecent theology sees an immediate link between structures of international oppression and heterosexual thinking in terms of hierarchical thought, ideological inflexibility and suppression of diversity.“

16 Vgl. Althaus-Reid / Isherwood, „Thinking Theology and Queer Theory,“ 311.

17 Vgl. Susannah Cornwall, ,,State of Mind“ versus ,Concrete Set of Facts': The Contrasting of Transgender and Intersex in Church Documents on Sexuality,“ Theology \& Sexuality, Bd. 15, 2015, $7-28,9$.

18 Auch Althaus-Reid hat exegetische Anstrengungen unternommen, um das queer-Paradigma bibeltheologisch zu verifizieren. So erinnert sie an die Erzählung von der Frau mit einem Blutsturz (Mt 9,18-22 par) und betont, Jesus habe mit keiner Silbe die herrschende Ideologie der Unreinheit der Frau wegen ihrer menses aufgegriffen, vgl. Althaus-Reid, From Feminist Theology, $14 \mathrm{f}$. $\mathrm{Zu}$ entsprechenden Themen in der Bibel vgl. für eine erste Übersicht Victoria S. Kolakowski, „Toward a Christian Ethical Response to Transsexual Persons,“ Theology \& Sexuality, Bd. 6, 1997, $10-31$, bes. $17-25$, und Fraser Watts, „Transsexualism and the Church,“ Theology \& Sexuality, Bd. 9, 2002, 63-85, 72f., 79f. Allerdings können aus solchen Beobachtungen schwerlich ethische Folgerungen gezogen werden, wie Falk Wagner betont, denn die Bibel sei geprägt durch ethische Widersprüche und intendiere kein universelles, ethisches System, vgl. Wagner, Zur Revolutionierung, 483.

19 Vgl. Elizabeth Stuart, „The Return of the Living Dead,“ in Post-Christian Feminisms, op. cit., 211 - 222, 219, und weiter Susannah Cornwall, „Apophasis and Ambiguity: The 'Unknowingness' of Transgender, “ in Trans/formations, hg. von Lisa Isherwood und Marcella Althaus-Reid, London: SCM 2009, 13 - 40, 38, wo sie zutreffend pointiert: „Human gender is important. However, it is not ultimate. Our gender identities are not the final word about us; they are part of our becoming but they are not the becoming itself." Kritisch dazu, weil es sich bei Paulus dabei lediglich um die geistliche Dimension des Glaubens handelt, die auf die christliche Gemeinde beschränkt ist, Harald Matern, ,....und schuf sie als Mann und Frau. Ethische Herausforderungen transidenter 
dieser weit ausgreifenden Insistenz auf Abkehr von einer bestimmten Façon maskulin-heteroprivilegierender Theologie ist „[t]he search for an alternative society based on principles of peace and justice [which] requires a radical theology that can allow alternative thinking“20. Dabei ist diese Art Kritik an einer Theologie, die Vielfalt und Andersheit dämonisiert, sehr heterogen, so dass Althaus-Reis das Label Queer Theology als umbrella term einführt. ${ }^{21}$ Neben dem Hinweis auf die „ambivalence and fluidity of sexual identities“ erscheint die damit zusammenhängende Annahme der Konstruktivität des Sexuellen nach Althaus-Reid gemeinsames Anliegen der Queer Theory, für die es Menschsein nicht anders als im Plural gibt. ${ }^{22}$ An das Menschsein plenis coloribus möchte diese Theologie erinnern und es (hermeneutisch) zur Geltung bringen.

Der konstitutive Praxisbezug einer Queer Theology motiviert Marcella-Reids klare Ortsbestimmung der Queer Theology an den Rändern der Gesellschaft, auch denen der Theologie. ${ }^{23}$ Dieses Merkmal der Queer Theology folgt zwar dem berechtigten Anliegen einer Pluralisierung innerhalb der Theologie, allerdings ist dabei fraglich, ob sie notwendig auf die Differentiale margin und centre angewiesen ist, wie Althaus-Reid insinuiert, oder ob nicht auch in einer Mitte Raum für Pluralität ist, die in einem so umgreifenden Sinne Diversität ermöglicht, dass der Unterschied zwischen Rändern und Mitte hinfällig wird. Es wäre daher zu fragen, ob die betonte Zuweisung an den Rand der Gesellschaft und die mitausgesagte Ausschließlichkeit nicht zugunsten eines inklusiven Ansatzes zu entschärfen wäre, der Menschsein überhaupt nicht als minorisierte Rand-Existenz toleriert. Althaus-Reids Lokalisierung der Queer Theology vermag auch deshalb nur bedingt zu überzeugen, weil die für sie unerwünschte Akzeptanz von der Mitte keineswegs unerheblich ist und dem sozialphilosophischen Paradigma der Gesellschaft als Anerkennungsgemeinschaft widerspricht, wie es unten mit Falk Wagner aufgegriffen wird (siehe II.1.).Von daher scheint es keineswegs erforderlich, wie AlthausReid meint, dass Queer Theology notwendig autobiographisches Format haben

Individualität aus theologischer Perspektive, “ in Inter* und Trans*identitäten. Ethische, soziale und juristische Aspekte, op. cit., 135-154, 147.

20 Althaus-Reid, „The Bi/girl Writing,“ 107.

21 Vgl. Althaus-Reid / Isherwood, „Thinking Theology and Queer Theory,“ 305 - 307.

22 Vgl. ebd.

23 Vgl. a.a.O., 304: „Terrible is the fate of theologies from the margin when they want to be accepted by the centre! Queer Theology strives, instead, for differentiation and plurality. Queer Theology is in this sense equivalent to a call for biodiversity in theology, that is, life and love in all its diversity“. 
müsse. ${ }^{24}$ Das Grundmotiv, die Würdigung sexueller und geschlechtlicher Vielfalt und damit einhergehend die Enttarnung einer bloß mit Schablonen argumentierenden Anthropologie des Geschlechtlichen, stellt ein allgemeines ethisches Interesse zum Schutz des gesellschaftskonstitutiven Individuums dar. ${ }^{25}$

\section{Queer Theology bei Cornwall}

Nach Susannah Cornwall ist queerness insofern in den biblischen Schriften und der Geschichte der christlichen Kirche virulent, als es bei näherem Zusehen dort entdeckt werden könne. ${ }^{26}$ Dabei bezieht sie sich auf die Vorgängerstudien von Marcella Althaus-Reid, die betont hatte, Gott sei deshalb queer, weil er mit seinem Volk ins Exil gegangen, also an die Ränder und so in die Vielfalt und Niederung menschlichen Schicksals herabgestiegen sei. ${ }^{27}$ Unmissverständlich habe AlthausReid dann betont, so Cornwall, nach der universellen Solidarisierung Gottes mit den Menschen dürfe es nicht schwerfallen, „to reflect on God as faggot and whore, as [...] pornographic and suicidal“ ${ }^{\text {228 }}$. Doch der eigentliche Punkt, so Cornwall, sei Althaus-Reids Plädoyer, Gottes Andersheit umfänglich zu begreifen und nicht notorisch dem bürgerlichen Lebensskript anzupassen. Die Entkoppelung des Gottesbegriffs von traditionellen Familienwerten hält Cornwall für gleichermaßen verstörend und effektiv. Effektiv deshalb, weil dem Gedanken der Inkarnation und der zum Ausdruck kommenden Fleischlichkeit Gottes das Verstörende zurückgegeben werde, das ein allzu kleinbürgerliches Material, aus dem sonst Gottesbilder gewoben seien, verharmlose. ${ }^{29}$ Althaus-Reids zuweilen vulgäre und verstörende Theologie ist natürlich nicht ohne Widerspruch geblieben, wie Cornwall betont. Trotz des ethischen Grundtenors ihrer Arbeit fallen intrikate theologische Probleme leicht ins Auge: Gott, der in jedem menschlichen Antlitz begegnet, könne nur als apophatischer Gott gedacht werden, der sich allen Klischees, dann

24 Vgl. ebd. Allerdings ist nicht zu bestreiten, dass eine Solidarisierung mit marginalisierten Gruppen in Wort und Tat zu einer Co-Marginalisierung führen kann, wie Althaus-Reid hervorgehoben hat, vgl. Althaus-Reid, „The Bi/girl Writing,“ 105.

25 Vgl. Wirth, „Was bedeutet: Unbedingte Anerkennung der Andersheit des Anderen?,“ 121123.

26 Vgl. Cornwall, Controversies, 147 und Wirth, „Living in a Shell,“ 1588 (Anm. 4).

27 Vgl. Marcella Althaus-Reid, The Queer God, London und New York: Routledge 2003, 146.

28 Cornwall, Controversies, 148.

29 Vgl. a.a.O., $148 \mathrm{f}$. 
aber auch denen der Queer Theology, entzieht. ${ }^{30}$ Leicht komme außerdem der Verdacht auf, Gott werde anthropomorph dargestellt, zumal bei hyperbolischer Sicht auf Sexualität. ${ }^{31}$ Problematisch sei auch das Interesse der Queer Theology an einer Fragmentierung menschlicher Identitäten und Gesellschaften, wie sie bei Althaus-Reid begegne, da so der Gottesbegriff um seinen Einheits- und Vereinigungswillen verkürzt würde. ${ }^{32}$

Integrativ fällt Cornwalls eigene Beantwortung der Frage aus, ob queer oder nicht-queer ein adäquates Label für die christliche Tradition abgibt, denn sie spricht beiden Hinsichten Deutungspotential zu. ${ }^{33}$ Jedenfalls habe Queer Theology, die betont mehr sein müsse als Theologie von und für Homosexuelle, einen originär-argumentativen Beitrag zur Aufdeckung solcher Normen geleistet, die Zwang ausüben („coercive norms“) und religiös legitimiert oder sogar produziert werden; ${ }^{34}$ auch im Sinne einer Konstruktion durch ritualisierte Wiederholung. ${ }^{35}$ Insofern verteidigt Cornwall Queer Theology gegen den Vorwurf theologischer Destruktivität und ethischer Bedeutungslosigkeit. Durch manche polemische Note oder bewusste Einseitigkeit wird ein ethischer Impuls gerade nicht ausgeschlos-

30 Negative Theologie und Theorien des Überschreitens und Entziehens implizieren einen Sinngehalt, den Cornwall nutzt, um Transidentitäten theologisch (in einem nicht-sensationellen Sinn) einzuordnen, vgl. Cornwall, „Apophasis and Ambiguity,“ $16 \mathrm{f}$.

31 Vgl. Cornwall, Controversies, $151 \mathrm{f}$.

32 Vgl. ebd. Eine entsprechende Kritik hat insbesondere Jeremy Carrette vorgetragen, dessen Wortmeldung allerdings selbst Resultat einer fehlgeleiteten Reflexion ist, die das konstitutive Plural der christlichen Theologie übersieht, wie es etwa im Trinitätsglauben Ausdruck gefunden hat, und exklusiv statt inklusiv argumentiert, vgl. Jeremy Carrette, „Beyond Theology and Sexuality: Foucault, the Self and the Que(e)rying of Monotheistic Truth," in Michel Foucault and Theology. The Politics of Religious Experience, hg. von Jeremy Carrette und James W. Bernauer, Aldershot: Ashgate 2004, 217 -232, 225: „Religion becomes queer when it breaks up the desiring self, when it refuses to confess an identity, when it refuses to say who we are, and acknowledges a plural self with polymorphous desires. To queer religion is to queer the foundations of theology, its monotheism, its monosexuality and its monopoly of truth." Kritisch dazu Susannah Cornwall, Sex and Uncertainty in the Body of Christ. Intersex Conditions and Christian Theology, London: Equinox 2010, 235, und weiter Eberhard Jüngel, „Die Wahrnehmung des Anderen in der Perspektive des christlichen Glaubens, “ in Indikative der Gnade - Imperative der Freiheit. Theologische Erörterungen IV, Tübingen: Mohr Siebeck 2000, 205-230, 206-213, der in alteritätstheoretischer Hinsicht die Pluralität des Monotheismus aufzeigt.

33 Vgl. Cornwall, Controversies, 186.

34 Vgl. Karle, „Da ist nicht mehr Mann noch Frau...“, 255 sowie Heather Looy und Hessel Bouma III, „The Nature of Gender: Gender Identity in Persons Who Are Intersexed or Transgendered,“ Journal of Psychology and Theology, Bd. 33, 2005, 166-178, 166.

35 Vgl. Wolfgang Detel, „Ein wenig ,Sex‘ muss sein. Zum Problem der Referenz auf die Geschlechter,“ Deutsche Zeitschrift für Philosophie, Bd. 45, 1997, 63-98, 69. 
sen, sondern offengelegt. ${ }^{36}$ Mit ihrer schonungslosen Offenlegung theologischer Einseitigkeiten („Queer theology does not allow any rug to be left unturned“37) ist Queer Theology zugleich konstruktiv, weil sie besonders am Beispiel des Geschlechtlichen und der Sexualität, einem kulturell stark umkämpften Terrain, exemplifiziert, wie selten Theologie konkrete Diversität menschlicher Existenz reflektiert. ${ }^{38}$ Eine Queer Theology, die nicht den Schneid hätte, gerade das Anthropologikum notorischen Andersseins in der Domäne des Sexus zu konkretisieren, würde notwendig zu harmlos ausfallen. Denn gerade hier erweist sie ihre Kraft, gegen das Vorurteil eines Geschlechts-Essentialismus ${ }^{39}$ und gegen die Monotonie des Körperlichen (Intersex) und Leiblichen (Trans) ${ }^{40}$ nichts als das Individuum zu sehen, selbst wenn es in den Augen des Establishments eine aura of strangeness umgibt. Wer schon die Vielfalt und Veränderbarkeit der brachialmaterialen Seite menschlicher Existenz normativ überspielt, wird schwerlich einen Zugang zu gedanklichen Pluralitäten finden; daher erscheint der Einsatz beim Sexus für eine Theorie der Anerkennung von Andersheit besonders plausibel. Notwendig und mithin ethisch gehaltvoll erscheint Cornwall eine solche Theologie auch angesichts der betrüblichen Tatsache, dass die Suizidalität insbesondere junger LGBTIs erhöht ist. Es gibt wichtige Hinweise, dass dies mit der unauffälligen, aber umso wirksameren Diktatur religiöser Ausgrenzungsmechanismen assoziiert ist, die LGBTIs als unerwünscht, illegitim und sündhaft darstellen. ${ }^{41}$ Als Echo auf persönliche Dramen queerer Biographien im Raum des Christentums hat Cornwall das Bild der Vuvuzela vorgeschlagen, was für LGBTIs in den christlichen Kirchen bedeutet, „to communicate manifestation, existence, identity, thereness“42.

36 Vgl. Cornwall, Controversies, 229.

37 A.a.O., 252.

38 Vgl. a.a.O., $252 \mathrm{f}$.

39 Vgl. Detel, „Ein wenig ,Sex‘, “ 68-74, und zur Kritik des Anti-Essentialismus, etwa bei Martha Nussbaum, vgl. a.a.O., $71-74$.

40 Vgl. Fehige, „Transsexualität,“ 762.

41 Vgl. Cornwall, Controversies, 254f. und Wirth, „Living in a Shell,“ 1587.

42 Cornwall, Controversies, 254. 


\section{Il Transidentität als theologische Nicht-Sensation: Theologie der Freiheit und Anerkennung}

Die innerhalb des Clusters Queer Theology elaborierten Näherbestimmungen der Vielfalt finden sich zwar weniger anschaulich, aber ebenso prägnant in Entwürfen der neueren theologischen Systematik dargestellt, die nach der Relevanz von Freiheit und Anerkennung fragen, wobei es zu einem nicht unerheblichen Allgemeinplatz geworden zu sein scheint, dass Ersteres ohne Letzteres nicht sein kann. ${ }^{43}$ Die theologische Überzeugung von der Anerkennung durch Gott wäre weltfremde und fromme Phantasie, wenn sie ohne Inkarnation bliebe: „Die Nagelprobe der aus der göttlichen Anerkennung resultierenden Gewißheit der Freiheit besteht darin, die Freiheit dadurch anzuerkennen, daß sie in die Praxis des täglichen Lebens übersetzt wird.“44 Eine solche Übersetzung hat die Queer Theology für den Bereich der LGBTIs begonnen. Ihre Auskünfte beziehen sich bisher selten auf große systematisch-theologische Entwürfe der Gegenwart, weshalb wichtige Denkmittel unbeachtet geblieben sind. ${ }^{45}$ Diese könnten aber maßgeblich für eine transpositive Theologie sein, da gegen allgemeine Anerkennungstheoreme der Theologie nicht so leicht der Verdacht der (notgedrungenen) Komplexitätsreduzierung geäußert oder Illegitimitätsvorwürfe laut werden.

\section{Falk Wagner und theologische Anerkennungsverhältnisse}

Folgt man der Befundung von Ulrich Barth, kann das Spätwerk Wagners als „Theologie der Anerkennung“ zusammengefasst werden. ${ }^{46}$ Die sozialethische Wende seiner Theologie ist dabei nicht unverbunden mit seiner durch Hegel inspirierten Arbeit am Gottesbegriff. Die binären Gottes- und Menschenbilder „Macht und Ohnmacht“, „Herrschaft und Knechtschaft“ sowie „Freiheit und Gehorsam“ werden von Wagner dementiert: Zum einen aus biblischen Gründen,

43 Vgl. Wagner, Zur Revolutionierung, 589.

44 A.a.O., 596f.

45 Vgl. Matern, ,...und schuf sie,“ 143 und Wirth, „Was bedeutet: Unbedingte Anerkennung der Andersheit des Anderen?,“ $123 \mathrm{f}$.

46 Vgl. Ulrich Barth, „Von der spekulativen Theologie zum soziologischen Religionsbegriff. Versuch einer Annäherung an das Denken Falk Wagners, “ Wiener Jahrbuch für Theologie, Bd. 3, 2000, 233-268, $255 \mathrm{f}$. 
weil Gott nicht als absolutes, unberührbares und unwandelbares Sein dargestellt wird, zum anderen aus logischen Gründen, denn die binäre Logik verlangt das eine zur Auslegung des anderen, wobei dann die Allmacht der Ohnmacht bedarf, was einen Widerspruch darstellt. ${ }^{47}$ Dieser Aporie entkommt der Gottesbegriff nach Wagner durch Komposition eines symmetrischen Gott-Mensch-Verhältnisses und nicht durch Vernichtung des Anderen: „Das aktive Bedingen der göttlichen Macht erweist sich zugleich als bedingt, und dem passiv bedingten Anderssein eignet zugleich die Bestimmung, bedingend zu sein [...]. Das göttliche Selbstsein und das welthaft-menschliche Anderssein bilden so ein symmetrisches-egalitäres Bestimmungsverhältnis“48. Die mitkonzipierte Verwiesenheit Gottes auf das Anderssein von Welt und Macht, die Wagner als „Revolutionierung des Gottesgedankens“ und aller Allmachtsphantasien präsentiert hat, ${ }^{49}$ schafft einen Raum für Anderssein und tatsächliches Selbstsein. Damit erhält der soziale Diskurs der Anerkennung konstitutive Bedeutung, denn nach Wagner kann es selbstständiges Anderssein nur unter der Voraussetzung geben, dass die Angewiesenheit auf selbstständiges Anderssein anerkannt wird: „[...] Jedes selbständige Selbstsein ist, um seine Selbständigkeit zu realisieren, auf ein selbständiges Anderssein angewiesen. Dieser Angewiesenheit lässt sich also nicht durch [...] Vernichtung des Andersseins Rechnung tragen. Vielmehr läßt sich das selbständige Selbstsein [...] nur dann realisieren, wenn sein selbständiges Selbstsein die Anerkennung des selbständigen Andersseins einschließt. “50 Selbstsein und Anderssein stehen also in einem reflexiven Verhältnis zueinander, nur in der Vermittlung des einen durch das andere, was Anerkennung bedeutet, bzw. „Selbstexplikation am Orte des Anderen seiner selbst“51, kann es für Wagner Freiheit geben. Absolute Selbstständigkeit, also das gänzliche Ausbleiben relationaler Anbindung, kann es in dieser Optik auch für Gott nicht geben. ${ }^{52}$

47 Vgl. Falk Wagner, Metamorphosen des modernen Protestantismus, Tübingen: Mohr Siebeck 1999, 150-152, und Falk Wagner, „Die christliche Revolutionierung des Gottesgedankens als Ende und Aufhebung menschlicher Opfer,“ in Zur Theorie des Opfers. Ein interdisziplinäres Gespräch, hg.von Richard Schenk, Stuttgart: Frommann-Holzboog 1995, 251 - 278, 270 - 272, dazu Mathias Wirth, Distanz des Gehorsams. Theorie, Ethik und Kritik einer Tugend, Tübingen: Mohr Siebeck 2016, $307-311$.

48 Wagner, Metamorphosen, 155f. Vgl. Barth, „Von der spekulativen Theologie,“ 260-262.

49 Vgl. Wagner, Metamorphosen, 158.

50 Ebd. Zu einer ähnlichen Argumentation in der Sozialphilosophie vgl. Wirth, „Was bedeutet: Unbedingte Anerkennung der Andersheit des Anderen?,“ 120-123.

51 Barth, „Von der spekulativen Theologie,“ 238.

52 Vgl. Wagner, Metamorphosen, 164-166, und Wagner, „Die christliche Revolutionierung,“ 154. Dazu Joachim Track, „Begriff und Thema der Religion,“ Theologische Rundschau, Bd. 56, 1991, 176-184. 
Die „Entsupranaturalisierung“ Gottes, die aus seinem Begriff selbst insofern folgt, als Gottes Macht nach Wagner nur aufrechtzuerhalten ist, wenn sie sich als Anerkennungsinstanz des Anderen erweist, ${ }^{53}$ thematisiert das positive Verhältnis verschiedener Freiheit, worin Wagner das Proprium der Religion in der Moderne sieht. ${ }^{54}$ Genauer wählt er die Begriffe Personalität (Selbstsein) und Sozialität (Anderssein), um der Religion der Moderne Bedeutung für die „Bearbeitung von Differenzerfahrungen“ zu verleihen (wie auch Kunst, Liebe, Psychotherapie etc.). ${ }^{55}$ Gegen den Trend zur bloß theoretischen Berücksichtigung individueller Freiheit, die ohne Wendung zur Materialität der Freiheit reine Ideologie bliebe, präzisiert Wagner das Verhältnis von Personalität und Individualität als Öffnung von Institutionen und Systemen für die einzelne Freiheit. ${ }^{56}$ Ethische Beurteilung nimmt dann Maß an Strukturen der Subjektivität, ${ }^{57}$ chancenlose Privatsachen gibt es dort nicht mehr, wo Anderssein priorisiert ist. ${ }^{58}$ Damit korreliert ein Freiheitsbegriff, den die Neuzeit als Selbstbestimmung auffasst („Privatisierung des Entscheidens ${ }^{\text {“59) }}$ ) und der die Fähigkeit beschreibt, etwas neu beginnen zu können. ${ }^{60}$

Es ist nicht schwer zu erkennen, dass die Entscheidung von Transpersonen vor diesem Befund nicht als Sensation, sondern als Folge der neuzeitlichen Weite konkreter Freiheit erscheinen muss, die Wagner keineswegs denunziert, sondern als schützenswert, anerkennungswürdig und zur theologischen Aufgabe erklärt: „Die Realisierung der auf Anerkennung basierenden Freiheit des Menschen ist die für die Grundidee des Christentums konstitutive Einsicht, mit der sie [...] steht und fällt.“61

53 Vgl. Wagner, Metamorphosen, 162, und dazu Wirth, Distanz des Gehorsams, 312.

54 Vgl. Wagner, Metamorphosen, 165.

55 Vgl. Martin Berger, „Krise und Zukunft der protestantischen Theologie in der Selektionsgesellschaft. Zu den Potentialen von Falk Wagners Theologiekritik, “ in Transformationsprozesse des Protestantismus. Zur Selbstreflexion einer christlichen Konfession an der Jahrtausendwende, hg. von dems. und Michael Murrmann-Kahl, Gütersloh: Chr. Kaiser 1999, 121-143, 126, und Wirth, Distanz des Gehorsams, 313.

56 Vgl. Wagner, Metamorphosen, 187 -190, und dazu Berger, „Krise und Zukunft,“ 123-127.

57 Vgl. Wagner, Zur Revolutionierung, 500.

58 Vgl. Berger, „Krise und Zukunft,“ 125.

59 Zitiert bei Barth, „Von der spekulativen Theologie,“ 243.

60 Vgl. Wagner, Zur Revolutionierung, 514.

61 A.a.O., 530; vgl. dazu Berger, „Krise und Zukunft,“ 139. 


\section{Eberhard Jüngel und theologische Anerkennungsverhältnisse}

Nach Jüngel fällt eine Trinitätstheologie zu harmlos aus, wenn sie die darin enthaltene zentrale und differenzempfindliche Pointe der Andersheit übergeht. Es sei gerade die trinitätsimmanente Struktur der Diversifikation, die die Gotteslehre präge, ${ }^{62}$ wobei diese konstitutive Bedeutung der Andersheit für die Anthropologie und schließlich die Ekklesiologie nicht unerheblich bleibe: ${ }^{63}$ „So wie Gott nur in personaler Gemeinschaft gegenseitigen Andersseins göttlich ist, so ist der Mensch nur menschlich, indem er als Person auf das Anderssein eines Anderen positiv bezogen ist. "“4 Diese analoge Rede, die göttliches und menschliches Anderssein in ein gewisses Entsprechungsverhältnis setzt, findet besonders im exzentrischen Charakter Gottes und des Menschen ihr tertium comparationis. Zwar handelt es sich bei Gott, so Jüngel im Anschluss an Karl Barth, um einen ewigen Entschluss, nicht anders sein zu wollen als in einem positiven Verhältnis zum Anderen, ${ }^{65}$ und beim Menschen eher um ein anthropologisches Faktum des notorischen Bezogenseins auf den Anderen. Trotzdem lässt sich für beide eine Art „Versenktheit in das Andere" beschreiben, ${ }^{66}$ das Gott konkretisiert und die Person erst konstituiert. ${ }^{67}$ Die religiöse Dimension dieses Exzesses ${ }^{68}$ auf das schlechthin Andere hin animiert nach Jüngel zu einer positiven Sichtung des Anderen, der sich theologisch nicht als zu bekämpfende Gefahr, ${ }^{69}$ sondern als „zugute kommendes Anderssein" erweise. ${ }^{70}$ Im Sinne der von Jüngel in der Rechtfertigungslehre bewusst umgekehrten Abfolge von Imperativ und Indikativ, die der Logik des konditionslosen Angenommenseins entspricht und eine gute Tat erst aus dem Indikativ

62 Gegen die Gefahr eines identitätslosen Zerfallens Gottes betont Jüngel die vornehmliche Einheit und Unveränderlichkeit Gottes, wie sie aus der dogmatischen Tradition folge, dennoch stellt er die Immanenz Gottes als „Verhältnis gegenseitigen Andersseins“ dar, vgl. Jüngel, „Die Wahrnehmung,“ 214-216.

63 Vgl. a.a.O., 205, und Peter Kline, „Participation in God and the Nature of Christian Community: Robert Jenson and Eberhard Jüngel,“ International Journal of Systematic Theology, Bd. 13, 2011, 38-61, 39 sowie Wirth, „Living in a Shell,“ 1593.

64 Jüngel, „Die Wahrnehmung,“ 217.

65 Vgl. a.a.O., 214.

66 Vgl. a.a.O., 217, und Wirth, „Was bedeutet: Unbedingte Anerkennung der Andersheit des Anderen?,“ 120.

67 Vgl. Eberhard Jüngel, „Zur Verankerung der Menschenrechte im christlichen Glauben,“ in Außer sich. Theologische Texte, Stuttgart: Radius 2011, 124-138, $136 \mathrm{f}$.

68 Vgl. Pröpper, Theologische Anthropologie, 501.

69 Vgl. Wirth, „Was bedeutet: Unbedingte Anerkennung der Andersheit des Anderen?,“ 114118.

70 Vgl. Jüngel, „Die Wahrnehmung,“ $217 \mathrm{f}$. 
des Angenommenseins erwächst, ${ }^{71}$ folgt entsprechend in seinem Alteritätskonzept aus dem Zugute-Kommen des Andersseins Gottes (Erbarmung, Rettung, Befreiung $)^{72}$ die Befähigung des Menschen zum Zugute-Kommen für den Anderen. Außerdem bezieht sich Jüngel auf Paulus und den Philipperbrief, der eine Ethik der Alterität enthält, die den Anderen um seiner selbst willen in Andersheit erhalten will (Phil 2,3f.). In der Priorisierung eines Anderen findet nach Jüngel aber keine „Sklavenmoral“ ihren Ausdruck, ${ }^{73}$ denn es gehe um die Aufgabe einer exklusiven Ego-Perspektive, die um die Alter-Perspektive zu erweitern sei. Sonst steht das Gute für den Anderen in der Gefahr, eigentlich nur das Gute für das Ich zu sein, was in die aporetische Situation führt, zu missachten, was man lieben könnte. ${ }^{74}$ In dieser sozialethischen Wendung des Trinitätsglaubens überwindet Jüngel auch den oft behaupteten Hiat zwischen Pflichten gegenüber Gott und Pflichten gegenüber Menschen. ${ }^{75}$ Dieser Konflikt führt im religiösen Ambiente im Umgang mit Trans dazu, unter Rekurs auf die Schöpfung und für sakrosankt genommene Genitalien ${ }^{76}$ die mögliche Pflicht gegenüber der Andersheit der Transperson gegen scheinbar widersprechende Pflichten vor Gott auszuspielen. ${ }^{77}$ Das hohe systematische Gewicht des Alteritätsdenkens verbietet nun aber offensichtlich, nicht-schadende Andersheit überwinden $\mathrm{zu}$ wollen, was bei Licht besehen bedeute, die Schöpfung insgesamt $\mathrm{zu}$ verneinen, die nicht uniform, sondern pluriform sei. Diese „hochkomplexe Vielfalt von Kreaturen“ gelte es „anzuerkennen und in Ehren zu halten“78. Daher gilt, und darin liegt eine besondere Pointe eines positiven Verhältnisses von Trans und Theologie, den Anderen in seiner Andersheit umfassend zu affirmieren, wie Jüngel gegen eine bloß aspekthafte Zuwendung (z. B. Almosengabe) betont, auch um die nicht zu dis-

71 Vgl. a.a.O., 220, und dazu Christoph Herbst, Freiheit aus Glauben. Studien zum Verhältnis eines soteriologischen Leitmotivs bei Wilhelm Herrmann, Rudolf Bultmann und Eberhard Jüngel, Berlin und Boston: De Gruyter 2012, 417 - 420, der auf den positiven Konnex von Freisein und Befreien in der Freiheitsanalyse Jüngels hinweist, um die Indikativ-Imperativ-Chronologie zu exemplifizieren.

72 Vgl. Ingolf U. Dalferth, „Gott für uns. Die Bedeutung des christologischen Dogmas für die christliche Theologie, “ in Denkwürdiges Geheimnis. Beiträge zur Gotteslehre. Festschrift für Eberhard Jüngel zum 70. Geburtstags, hg. von dems., Johannes Fischer und Hans-Peter Großhans, Tübingen: Mohr Siebeck 2004, 51-75, 73.

73 Vgl. Wirth, Distanz des Gehorsams, $323 \mathrm{f}$.

74 Vgl. Jüngel, „Die Wahrnehmung,“ $220 \mathrm{f}$.

75 Vgl. a.a.O., 222.

76 Vgl. Wirth, „Living in a Shell,“ 1591.

77 Vgl. a.a.O., $1590 \mathrm{f}$.

78 Eberhard Jüngel, „Mensch, wo bist Du? Glauben und Freiheit als Ortsbestimmungen des Christenmenschen,“ in Außer sich. Theologische Texte, op. cit., 13-40, 39. Vgl. Pröpper, Theologische Anthropologie, 153. 
pensierende Fleischlichkeit des Menschseins $\mathrm{zu}$ integrieren, die im Falle der Transidentität von besonderer Relevanz ist: ${ }^{79}$,sie [scil. die Nächstenliebe] nimmt den Anderen in seinem Anderssein wahr und bejaht ihn in seinem Anderssein. Sie identifiziert sich mit ihm, indem sie ihn gerade nicht vereinnahmt, nicht nostrifiziert, nicht um sein Anderssein betrügt. Sie konstituiert vielmehr - in strenger Analogie zum Sein des dreieinen Gottes - eine nun freilich menschliche Gemeinschaft gegenseitigen Andersseins.“80

Es ist kein Zufall oder eine Marginalie, wenn Transidentität hier auf die Alteritätskonzeption Jüngels bezogen wird. Da es sich bei Trans nicht um eine modische Extravaganz oder ein äußerlich bleibendes Makeover handelt - als ob man seine Geschlechtlichkeit abschleifen könnte wie Akne -, sondern um eine Frage existentiellen Sein-Könnens, fallen Zurückweisungen als Weigerung personaler Anerkennung aus. Jüngel betont in seiner Alteritätskonzeption den besonderen Fokus der Evangelien auf die Gruppe der Geschmähten, deren Status als Seliggepriesene nicht das Leid selbst ästhetisiert, sondern Schutz für das Anderssein des Trauernden, Ohnmächtigen, Gewaltlosen etc. einfordert. In diese Reihe lässt sich heute aufgrund virulenter Ausgrenzungsmechanismen das Schicksal vieler LGBTIs einordnen. Selig nenne der Jesus des Evangeliums, so Jüngel weiter, nicht nur die Diffamierten selbst, sondern auch die, die eine Alter-Perspektive einnehmen. ${ }^{81} \mathrm{Ob}$ man dies Mitleid nennen muss, ist eine andere Frage (Mt 5,7), jedenfalls erscheint Trans als theologisches Thema von Rang. Jüngel belässt den Hinweis zum Recht auf Berücksichtigung nicht im Pauschalen und votiert für eine Konkretion durch den von ihm mehrfach diskutierten Friedensbegriff. Dabei kommt es ihm darauf an, den vielfach beschworenen Frieden (schalom) als einen Modus des Zusammenlebens zu begreifen, in dem Anderssein und mithin Personsein möglich ist: ${ }^{82}$ „Wer Frieden macht, der gewährt dem anderen zumindest

79 Vgl. Eberhard Jüngel, „Ganzheitsbegriffe - in theologischer Perspektive,“ in Ganz werden. Theologische Erörterungen V, Tübingen: Mohr Siebeck 2003, 40-53, 46.

80 Jüngel, „Die Wahrnehmung,“ 223. Vgl. auch Gunther Wenz, „Von den Letzten Dingen. Eschatologische Perspektive,“ Kerygma und Dogma, Bd. 61, 2015, 362-386, 376: „Nächstenliebe anerkennt und fördert das Anderssein des anderen“. Vgl. auch Gunther Wenz, „Vom apostolischen Osterzeugnis. Notizen zu Gedanken Hans-Georg Geyers,“ in Wahrheit und Versöhnung. Theologische und philosophische Beiträge zur Gotteslehre, hg. von Dietrich Korsch und Hartmut Ruddies, Gütersloh: Gütersloher Verlagshaus 1989, 167 -189, 188: „Grundlegend für die institutionelle Verfassung der Kirche [...] muss der stetige Wille sein, einem solchen Zusammensein der Verschiedenen zu dienen, in dem Verschiedenheit, ohne zu trennen, als Verschiedenheit bewahrt bleibt und der einzelne oder die Gruppe nicht einem mehr oder minder gewaltsamen Identifikationszwang ausgesetzt werden.“

81 Vgl. Jüngel, „Die Wahrnehmung,“ 225.

82 Vgl. a.a.O., 226, und dazu Wirth, Distanz des Gehorsams, 321. 
ein Minimum an schöpferischer Geborgenheit, die es dem Anderen erlaubt, ohne Furcht aus sich herauszugehen und seinerseits beim Anderen zu sich selbst zu kommen. Ohne ein Minimum schöpferischer Geborgenheit kann kein Mensch im Frieden leben. ${ }^{\text {83 }}$ Besonders die Kirchen bestimmt er als entsprechenden Raum benevolenten Zusammenseins. ${ }^{84}$ Liturgisch finde dies Ausdruck im Friedensgruß, der „wie nichts in der Welt das Anderssein des Anderen gedeihen [lässt]. “85 Es bedarf keiner spekulativen Klimmzüge, um Jüngels explizites Votum für das Selbstsein des Menschen (,jede Kreatur [darf] sie selber sein“86) für die Transfrage zur Geltung zu bringen. Eine Praxis des Friedens bewährt sich erst dort, wo sie eine Sphäre der Offenheit für den Anderen kreiert, gerade wenn die in Rede stehende Andersheit quer zur sozialen Privilegierung des Üblichen steht. Schließlich bedeutet Frieden auch „elementare Existenzberuhigung“87 für die kontingente Sensationalisierung der Transperson.

Friede ist zugleich als Begrenzungsgeschehen $\mathrm{zu}$ verstehen und in diesem Sinn als Aufgabe für die Kirchen, ${ }^{88}$ eine letztlich bürgerlich inspirierte Vorstellung von Lebensskripten nicht zur Norm zu erheben und alle abweichenden Konzepte in einem ohnehin stets willkürlichen biblischen oder theologischen Eklektizismus zu diskriminieren.

Weite Teile der Theologie Jüngels widmen sich dem Christentum als Religion der Freiheit. ${ }^{89}$ Er hat viele Anstrengungen unternommen, Freiheit als Spitzensatz seines Entwurfs zu konkretisieren, in dem die (rechtfertigungstheologische) Überzeugung herrscht, nur die Person wirke Befreiung, die selbst Befreiung erfahren habe. ${ }^{90}$ Das gilt auch umgekehrt: Wo das Christentum nicht befreiend wirkt,

83 Jüngel, „Die Wahrnehmung,“ 229. Vgl. auch Eberhard Jüngel, „Zum Wesen des Friedens. Frieden als Kategorie theologischer Anthropologie, " in Ganz werden. Theologische Erörterungen V, op. cit., 1-39, 31.

84 Vgl. Jüngel, „Zur Verankerung,“ 135.

85 Jüngel, „Die Wahrnehmung,“ 230. Vgl. auch Jüngel, „Zum Wesen,“ 25 - 28, und dazu Wirth, „Living in a Shell,“ 1593.

86 Jüngel, „Die Wahrnehmung,“ 230.

87 Jüngel, „Zum Wesen,“ 26.

88 Vgl. Kline, „Participation in God,“ 54.

89 Vgl. Wirth, Distanz des Gehorsams, $317 \mathrm{f}$.

90 Vgl. Herbst, Freiheit aus Glauben, 427. Dieser befreiende Charakter des Christentums ist für Jüngel nicht zuletzt christologisch fundiert. Da man nach Jüngel nichts von Gott sagen könne, was nicht auch für Jesus gelten könnte (,Wer und was Gott ist, ist allererst in der Einheit Gottes mit Jesus bestimmbar“, Eberhard Jüngel, „Thesen zur Grundlegung der Christologie,“ in Unterwegs zur Sache. Theologische Bemerkungen, 3. Aufl., München: Kaiser 2000 [1972], 274 - 295, 276; vgl. dazu Dalferth, „Gott für uns, “ 51 sowie David F. Ford, „Hosting a Dialogue: Jüngel and Lévinas on God, Self and Language, “ in The Possibilities of Theology. Studies in the Theology of Eberhard Jüngel in his Sixtieth Year, hg. von John Webster, Edinburgh: T\&T Clark 1994, 23 -59), Jesus aber ent- 
degeneriert es zu einer bloßen Schrumpfform. Die brachial-materiale Freiheit zu Geschlechtlichkeit und Sexualität mit rigider Zurückweisung traktieren bedeutet in diesem Sinn, den Glauben an den befreienden Gott aufzugeben. Es ist nach Jüngels Theologie aber keineswegs gottesfürchtig, fromm oder kirchlich, rigide Kultureme des Geschlechtlichen oder Sexuellen mit dürftigen biblischen und theologischen Meinungen oder dem unmöglich gewordenen Rekurs auf eine ursprüngliche Bestimmung des Menschen immer weiter zu wiederholen. ${ }^{91}$

\section{Transidentität als Ernstfall Systematischer Theologie? (Fazit)}

Zwar verliert die Zurückweisung der Andersheit des Anderen jeden vertretbaren Sinn, weil es sich bei Licht besehen stets um eine Annihilation des Menschen handelt, der niemals einer Man-Instanz zu entsprechen vermag. ${ }^{92} \mathrm{Ob}$ aber dem konkreten Anderssein sowohl politisch als auch gesellschaftlich die Zukunft gehört, wird davon abhängen, das negative Präjudiz, etwa gegenüber den Ambiguitäten des Sexuellen und Geschlechtlichen, abzubauen. Dass es sich dabei um ein edles, aber entfernt liegendes Ziel handelt, ist kaum zu leugnen. Trans wird zum Beispiel immer noch von pathozentrischen Vorannahmen begleitet, ${ }^{93}$ als sozial abweichend empfunden und sogar in theoretischen Diskursen mit dem Vorwurf konfrontiert, die Kultureme des Mann- und Frauseins mit allen Vorurteilen auch noch $\mathrm{zu}$ vertiefen. ${ }^{94}$

schiedenes Freiheitshandeln charakterisiert, auch und gerade gegen die Konventionen des Establishments, ist Christentum ohne eine entsprechende Priorisierung gründlich missverstanden, vgl. Pröpper, Theologische Anthropologie, 496: „Er [scil. Jesus] hat die Freiheit anerkannt, die er suchte, und indem er sie anerkannte, befreite er sie.“

91 Vgl. Striet, „Fortschritt und Kontingenz,“ $131 \mathrm{f}$.

92 Vgl. Cornwall, Sex and Uncertainty, 230 und Mathias Wirth, „Psychiatrie und Freiheit. Geschichte und Ethik einer Menschheitsfrage,“ Kirche und Gesellschaft, Nr. 398, 2013, 3-16.

93 Vgl. Fehige, „Transsexualität,“ 763.

94 Vgl. als Beispiele Detel, „Ein wenig ,Sex“, “ 93, und Fehige, „Transsexualität,“ 759. Dabei wird einerseits völlig übersehen, dass man den in heterosexuellen Verhaltensmustern aufgehenden Personen auch nicht den Vorwurf macht, kein Abbild des queer-Paradigmas zu liefern. Problematisch würde es doch erst dann, wenn dies mit dem Gestus der Normativität verbunden wäre. Wieso sollte dann von Transpersonen verlangt werden, sich nicht in bestimmten Geschlechterrollen einzurichten? Andererseits ist das Argument auch auf theoretischer Ebene inkonsistent, denn gerade die Transition zwischen den Geschlechtern macht doch gerade das Poröse deutlich und erscheint somit notwendig als Falsifizierung eines Geschlechts-Essentialismus. Außerdem stimmt das Argument Detels und Fehiges schon empirisch nicht, weil nicht alle Transpersonen 
Dabei erweisen sich Trans-Biographien in gewisser Hinsicht als anthropologische Schule. Trans erscheint nämlich als fulminantes Beispiel der Distanzierungsfähigkeit der Person gegenüber der Wirklichkeit des eigenen Körpers. ${ }^{95}$ Solche Distanzfähigkeit erscheint geradewegs als kulturelles Potential des Menschen. Es scheint, dass Religion und Wissenschaft darin ein Gemeinsames haben, ein „Gehäuse“ bereitzustellen, mit dessen Hilfe Menschen die immer auch nötige Distanz zur Wirklichkeit dauerhaft wahren können. ${ }^{96}$ Insofern stellt das Phänomen der Transidentität keinesfalls einen Fall sui generis dar, sondern erscheint als besonders frappante Variante des Distanzierens, die ebenfalls einen Ort im genannten Gehäuse von Religion und Wissenschaft hat.

In Theologie und Kirche ist aber eine Erfahrung der Erfahrungslosigkeit mit Transidentität und vielen Formen des konkreten Andersseins unübersehbar; bis auf wenige Ausnahmen finden sich keine Thematisierung und erst recht keine Antworten. ${ }^{97}$ Dass insbesondere die Systematische Theologie erhebliches Potential $\mathrm{zu}$ einer nicht-sensationellen Würdigung von Andersheit hat, sollte hier aufgewiesen werden. Eine das Paradigma der Anerkennung anführende Theologie würde überdies inklusiv und nicht exklusiv argumentieren und lieferte Denkmittel „zur Schaffung eines genauen, humanen und genuin befreienden Gedankenkorpus über die Sexualität"98. Jedes andere theologische Votum verlöre vor dem christlich-ethischen Anspruch des unbedingten Anspruchs des Anderen jede Legitimation. ${ }^{99}$ Gegen eine Theologie der Unwissenheit, Verdrängung und Un-

geschlechtsangleichende Operationen vornehmen lassen, also nicht notwendig in einem umfassenden Sinn im neuen Geschlecht aufgehen, vgl. Lindemann, Das paradoxe Geschlecht, 294296; Looy / Bouma III, „The Nature of Gender,“ 168 und Cornwall, „Apophasis and Ambiguity,“ $37 \mathrm{f} .:$,Transgendered people often desire a clear expression of gender which is unambiguously and unremarkably understood by others. In this they are far from alone." Hieran knüpft sich außerdem die Frage, ob es nicht ein rigides dichotom-geschlechtliches Denken einer Gesellschaft ist, das nicht nur Transpersonen eine distinkte Performanz des Geschlechtlichen abverlangt, vgl. Kolakowski, „Toward a Christian Ethical Response,“ 22.

95 Vgl. die obigen Ausführungen zu Thomas Pröppers Begriff der formal unbedingten Freiheit (Anm. 8).

96 In Anlehnung an Hans Blumenberg hat dies Martin Zerrath, Vollendung und Neuzeit. Transformation der Eschatologie bei Blumenberg und Hirsch, Leipzig: Ev. Verlagsanstalt 2011, ausgearbeitet.

97 Vgl. Matern, ,....und schuf sie,“ 141, und Cornwall, „State of Mind,“ 23-25.

98 Gayles S. Rubin, „Sex denken. Anmerkungen zu einer radikalen Theorie der sexuellen Politik,“ in Queer Denken. Gegen die Ordnung der Sexualität, hg. von Andreas Kraß, Frankfurt am Main: Suhrkamp 2003, 31-79, 32.

99 Was ist zum Beispiel mit dem Grundgebot der Gastlichkeit, die jedem bedingungslos geschuldet ist und voraussetzt, dem Anderen Raum zu gewähren? Vgl. Karle, „Da ist nicht mehr Mann noch Frau...“, 258; Looy / Bouma III, „The Nature of Gender,“ 174; und Burkhard Liebsch, 
terdrückung sondiert eine Anerkennungs-Theologie die nicht selbstverständliche „Liebe über alle Maßen“, ohne zu verletzen, ohne zu quälen, geradezu behutsam.

„Spielräume einer Kultur der Gastlichkeit. Zwischen Ethik, Recht und Politik“ (Teil 2), Philosophische Rundschau, Bd. 62, 2015, 243-260. 